\title{
An illustration of delays in Mucormycosis: A case Study
}

\section{Neelam Anupama Toppo ${ }^{1}$, Aditya Thakur ${ }^{2}$, Deepali Soni ${ }^{3}$, Priyanka Dubey ${ }^{4}$, Sapna Tiwari ${ }^{5}$}

${ }^{1}$ Professor, Department of Community Medicine, Netaji Subhash Chandra Bose Medical College, Tilwara Road, Garha Purwa, Jabalpur, Madhya Pradesh; ${ }^{2}$ Associate Professor, Department of Community Medicine, Netaji Subhash Chandra Bose Medical College, Tilwara Road, Garha Purwa, Jabalpur, Madhya Pradesh; ${ }^{3}$ Post Graduate Residents, Department of Community Medicine, Netaji Subhash Chandra Bose Medical College, Tilwara Road, Garha Purwa, Jabalpur, Madhya Pradesh; ${ }^{4}$ Post Graduate Residents, Department of Community Medicine, Netaji Subhash Chandra Bose Medical College, Tilwara Road, Garha Purwa, Jabalpur, Madhya Pradesh; ${ }^{5}$ Post Graduate Residents, Department of Community Medicine, Netaji Subhash Chandra Bose Medical College, Tilwara Road, Garha Purwa, Jabalpur, Madhya Pradesh

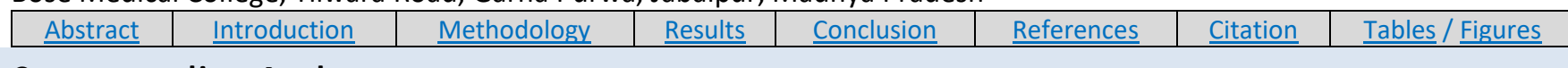

\section{Corresponding Author}

Dr Neelam Anupama Toppo, Professor, Department of Community Medicine, Netaji Subhash Chandra Bose Medical College, Jabalpur, Madhya Pradesh - 482003

E Mail ID: neelam.philips2011@gmail.com

\section{Citation}

Toppo NA, Thakur A, Soni D, Dubey P, Tiwari S. An illustration of delays in Mucormycosis: A case Study.Indian J Comm Health. 2021;33(3):515-518. https://doi.org/10.47203/IJCH.2021.v33i03.019

Source of Funding: Nil Conflict of Interest: None declared

\section{Article Cycle}

Received: 01/06/2021; Revision: 27/07/2021; Accepted:21/08/2021; Published:30/09/2021

This work is licensed under a Creative Commons Attribution 4.0 International License.

\section{Abstract}

Background: Mucormycosis is an opportunistic fungal infection with a high fatality rate and is the third most common fungal infection that is invasive in nature, next to candidiasis and aspergillosis. It is attributed to the poor socio-economic status and triggered by the local trauma due to unhygienic setup or poor health care. The pathway of pathogenesis is not clearly understood in immunocompetent patients and therefore becomes a matter of great concern.Aim\& Objective: To explore the pathway of mucormycosis in a case of post SARS-CoV-2 infection Settings and Design: Tertiary Care hospital of Jabalpur DistrictMethods and Material: Interview with the case and care taker and case file review.Results: We could conclude this as a case of rhino- orbital mucormycosis attributed to uncontrolled blood sugar during treatment of SARS-CoV-2 infection, three delays that have occurred during the disease progression like ignorance from health service provider, late diagnosis, non compliance to the advice given by health facility, poor oral and nasal hygiene and no use of distilled water in humidifier. Conclusions: The early diagnosis, prompt Treatment, surgical intervention, proper blood sugar monitoring and rational use of steroids are important steps of successful outcome of the disease.

\section{Keywords}

Mucormycosis; COVID-19; Delivery of Health Care; Fungal infection and uncontrolled diabetes

\section{Introduction}

Mucormycosis is a rare, opportunistic, aggressive, fulminant, and frequently fatal infection, contributing to $8.3 \%-13.0 \%$ of all fungal infections. Reported by Paltauf in 1885 and R.D. Baker coined the term Mucormycosis later. (1)

Predisposing factors reported are AIDS, uncontrolled diabetes mellitus, cancers such as lymphomas, kidney failure, organ transplant, long term corticosteroid and immunosuppressive therapy, neutropenia, iron overload or hemochromatosis. (2)

SARS-CoV-2 infection has played havoc with millions of lives worldwide. In this highly contagious disease, supportive care plays a pivotal role in reducing the mortality and morbidity due to SARS-CoV-2.

Though Corticosteroids have shown promising results in the management of COVID-19, but other side they increase the risk of secondary infections too. The risk of opportunistic infections in COVID-19 patients is further enhanced by the immune dysregulation caused by the virus and the use of concurrent steroid drugs and immune-modulatory drugs such as tocilizumab.(3)Mucormycosis is one such fungal infection which has emerged as a matter of concern in SARS-CoV-2 infection, as the predisposing factors for both are the same. The use of corticosteroids to treat severe/critical COVID-19 is a well-known risk factor for Mucormycosis. (4) 
In India during second wave of COVID pandemic there has been sudden emergence of Mucormycosis cases. In central India also cases being reported and was declared as Epidemic. This case study was one among those early diagnosed cases of Mucormycosis.

\section{Aims \& Objectives}

To explore the pathway of mucormycosis in a case of post SARS-CoV-2 infection

\section{CASE DESCRIPTION}

A 57 years male found positive for COVID-19 on 20th April 2021 at Batiyagarh village of District Damoh. On 22nd April HRCT chest was done and found CT severity score of $12 / 25$. He came to District Katni on 23rd April and got admitted in District hospital and treatment started. He was known case of Diabetes Mellitus since 20 years. Till 28th April he got treatment for COVID-19 at District hospital where he was given, Antibiotics, Steroids, Low molecular weight Heparin, Remdesivir, oral vitamin and mineral supplementation. During the treatment he was kept on Oxygen support. His blood sugar was not controlled adequately inspite of giving Insulin. His blood sugar level was always 200 to $250 \mathrm{mg} / \mathrm{dl}$ and raised up to $572 \mathrm{mg} / \mathrm{dl}$. On 4th day of admission, he reported pain and swelling on the right side of his nose and cheek area. As on this day case was stable with his oxygen saturation, he was shifted to COVID Care Centre as stepped down process. Though symptoms of Mucormycosis was gradually increasing and was shared to local clinician but was ignored as were told that it will be alright with antibiotics and analgesics. But his problem was continuously increasing with swelling and pain on right side of his face as well his blood sugar reached up to 572 $\mathrm{mg} / \mathrm{dl}$. Then case was again shifted again to District hospital. Here with the suspicion of Mucormycosis he was asked to refer to higher center i.e., Medical college Jabalpur on 28thApril. But patient didn't reach to Medical college rather visited private Ophthalmologist, Neuro surgeon and local traditional healer. Initially he took treatment from traditional healer for his symptoms but could not be relieved. Then he visited to ophthalmologist and on 3rd May he was found with Right Eyes Ptosis, diminution of vision in same eye and Right maxillary swelling as well blackish ulcer on hard palate. (Figure 1) On 9thmay MRI PNS was done and found with mucosal thickening and hyper intensity and air fluid levels in right ethmoid and sphenoid sinus [acute sinusitis], Mild mucosal thickening in right maxillary sinus causing partial opacification [sinusitis] and Right orbital and periorbital cellulitis with inflammatory changes in soft tissues of right face.

On 9th may his eye ball movement was also stopped. He got admitted in private hospital in Jabalpur and his treatment with Amphotericin B was initiated. The patient was scheduled for Eye surgery but found Covid positive again in RTPCR test so referred to Medical College,
Jabalpur. In Medical college patient undergone for debridement surgery. And still he is in hospital and getting treatment of inj.meropenem, liposomal amphotericin B $1.5 \mathrm{mg} / \mathrm{kg}$ body wt. ( Figure 2)

\section{Discussion}

It was the case of Rhino-orbital (sinus and eyes) mucormycosis where One-sided facial swelling, pain in right side of nose, headache, blackish lesions on hard palate of mouth, diminution of vision with Ptosis was there. He was known case of uncontrolled diabetes mellitus since 20 years. A case study performed on 60 year male with long standing diabetes mellitus with similar treatment history of parenteral meropenem, oral oseltamivir with parenteral methylprednisolone and a single dose of injectable tocilizumab. And during his course of treatment at hospital he developed signs of orbital cellulitis and nasal biopsy revealed broad aseptate filamentous fungal hyphae suggested of mucormycosis, which was confirmed on culture. (5)

A study from New Delhi, India on 15 admitted patients with SARS-CoV-2 infection reported bloodstream candida infections. Of these, 10 had a Candida auris infection, of whom six died (60\%).(6) P. Lewis White et.al found strong association between patients with multiple Aspergillus/BDG $(\geq 2)$ positive results and the use of highdose systemic corticosteroids. The use of corticosteroids (P: 0.007) and history of chronic respiratory disease ( $P$ : 0.05 ) increased the likelihood of aspergillosis. (7)

The immune dysregulation associated with COVID-19, with reduced numbers of $\mathrm{T}$ lymphocytes, $\mathrm{CD} 4+\mathrm{T}$, and $\mathrm{CD} 8+\mathrm{T}$ cells, may alter innate immunity, administration of corticosteroids as line of treatment also act as a catalyst which may permit and facilitate secondary fungal infections.

Aditya Moorthy et al found 16 /18 patients were diabetic and of those 15 received steroids for Covid-19 treatment. They reported Loss of vision in 12 cases and most of the cases were noted with mucormycosisi.e. 16/18 patients, aspergillosis in one patient and a mixed fungal infection in 1 patient. (8)

According to another study done by S. Sharma et al, reported cases of Mucormycosis as a fungal infection mostly associated with coronavirus disease 2019. Similar with our case, they also found sinuses being involved were the ethmoids followed by the maxillary sinus. They also found Diabetes mellitus as predisposing factor for Mucormycosis. It was documented that uncontrolled diabetes further increases the risk where intra-orbital involvement was found to be common but intracranial involvement occurs rarely. Extensive steroid and broadspectrum antibiotic use for Covid-19 management was found to be a cause or worsen the fungal disease in their study.(9)

As our country has been called the capital of Diabetes that could be reason for rise of Mucormycosis cases during this 
COVID -19 pandemic. It has also been found that SARSCoV-2 infection has been associated with altered glucose metabolism. Specifically, ketosis and ketoacidosis were observed during and after SARS-CoV-2 infection. Study suggest that human $\beta$-cells express viral entry proteins, and SARS-CoV-2 infects and replicates in cultured human islets. Which reduces numbers of insulin-secretory granules in $\beta$-cells and impaired glucose-stimulated insulin secretion ultimately impairing the glucose homoeostasis.(10)

During this pandemic corticosteroid found to be life-saver in Covid-19 cases, it has been now coming as a doubleedged sword where it was used irrationally. It is important and essential that this must be used with all caution and discretion. It has been understood that presence and uncontrolled diabetes, poor monitoring during treatment of COVID -19, poor oral and nasal hygiene made the case vulnerable for Mucormycosis and is an indicator of poor prognosis. maintaining good glycaemic control and early recognition vital for a favourable outcome.

\section{Conclusion}

In this case, the blood Sugar was not properly monitored or controlled by Insulin. Symptom of Mucormycosis started during hospital stay that was ignored by health workers or else further progression could be stopped by timely diagnosis and intervention of Antifungal drugs. This was found to be first delay with 24 hours. Disease further progressed at Covid Care Centre where patient's blood sugar raised upto $572 \mathrm{mg} / \mathrm{dl}$ and then re- admitted to the District Hospital shows blood sugar was not maintained adequately and no diagnosis made till date this was second delay with further 24 hours. Patient was asked to get admitted and take further treatment from higher centrei.e Medical college. He didn't come to Medical college rather visited many private doctors was third delay of 6 days. Nasal and Oral hygiene may not be maintained and distilled water was not used in humidifier during his COVID -19 treatment made case vulnerable for Mucormycosis.

\section{Recommendation}

During the treatment of SARS- CoV-2, blood glucose level must be monitored atleast three times a day and maintained within the limit with insulin or oral drugs, Oral and nasal hygiene must be maintained, Distilled water must be used in humidifier and early diagnosis and initiation of correct treatment is recommended.

\section{Relevance of the study}

As mucormycosis followed by SARS Cov-2 infection has been observed in our state during second wave of pandemic and this was the one among first cases with detail case study will help and strengthen the knowledge of epidemiologist, practitioners and policy makers.

\section{Authors Contribution}

All authors have contributed equally.

\section{References}

1. Ramalingam $S$, Narasimhan $M$, Vinithra $K$ and Vyshnavi JD, Mucormycosis : A Brief Review, J Pure Appl Microbiol., 2019; 13(1):161-165 doi: 10.22207/JPAM.13.1.16.

2. Centre for disease control and prevention [internet], Fungal Disease -Mucormycosisavailable from https://www.cdc.gov/fungal/diseases/mucormycosis/index.html[ Accessed on 28/05/2021]

3. Garg D, Muthu V, Sehgal IS, Ramachandran R, Kaur H, Bhalla A et al. Coronavirus Disease (Covid-19) Associated Mucormycosis (CAM): Case Report and Systematic Review of Literature. Mycopathologia. 2021 May;186(2):289-298. doi: 10.1007/s11046021-00528-2. Epub 2021 Feb 5. PMID: 33544266; PMCID: PMC7862973.

4. John TM, Jacob CN, Kontoyiannis DP. When Uncontrolled Diabetes Mellitus and Severe COVID-19 Converge: The Perfect Storm for Mucormycosis. J Fungi (Basel). 2021 Apr 15;7(4):298. doi: 10.3390/jof7040298. PMID: 33920755; PMCID: PMC8071133.

5. Mehta S, Pandey A. Rhino-Orbital Mucormycosis Associated With COVID-19. Cureus. 2020 Sep 30;12(9):e10726. doi: 10.7759/cureus.10726. PMID: 33145132; PMCID: PMC7599039.

6. Chowdhary A, Tarai B, Singh A, Sharma A. Multidrug-Resistant Candida auris Infections in Critically III Coronavirus Disease Patients, India, April-July 2020. Emerg Infect Dis. 2020 Nov;26(11):2694-2696. doi: 10.3201/eid2611.203504. Epub 2020 Aug 27. PMID: 32852265; PMCID: PMC7588547

7. White PL, Dhillon R, Cordey A, Hughes H, Faggian F, Soni S et al, A National Strategy to Diagnose Coronavirus Disease 2019Associated Invasive Fungal Disease in the Intensive Care Unit. Clin Infect Dis. 2021 Oct 5;73(7):e1634-e1644. doi: 10.1093/cid/ciaa1298. PMID: 32860682; PMCID: PMC7499527.

8. Moorthy A, Gaikwad R, Krishna S, Hegde R, Tripathi KK, Kale PG et al. SARS-CoV-2, Uncontrolled Diabetes and Corticosteroids-An Unholy Trinity in Invasive Fungal Infections of the Maxillofacial Region? A Retrospective, Multi-centric Analysis. J Maxillofac Oral Surg. 2021 Mar 6;20(3):1-8. doi: 10.1007/s12663-021-01532-1. Epub ahead of print. PMID: 33716414; PMCID: PMC7936599.

9. Sharma S, Grover M, Bhargava S, Samdani S, Kataria T. Post coronavirus disease mucormycosis: a deadly addition to the pandemic spectrum. J Laryngol Otol. 2021 May;135(5):442-447. doi: 10.1017/S0022215121000992. Epub 2021 Apr 8. PMID: 33827722; PMCID: PMC8060545.

10. Müller JA, Groß R, Conzelmann C, Krüger J, Merle U, Steinhart J et al. SARS-CoV-2 infects and replicates in cells of the human endocrine and exocrine pancreas. Nat Metab. 2021 Feb;3(2):149165. doi: 10.1038/s42255-021-00347-1. Epub 2021 Feb 3. PMID: 33536639 . 


\section{Figures}

FIGURE 1PROGRESSION OF THE SYMPTOMS [ORBITAL CELLULITIS, BLACKENING OF NOSE BRIDGE, PTOSIS IN RIGHT EYE, SWELLING OF RIGHT MAXILLARY AREA, NO MOVEMENT OF RIGHT EYE BALL]

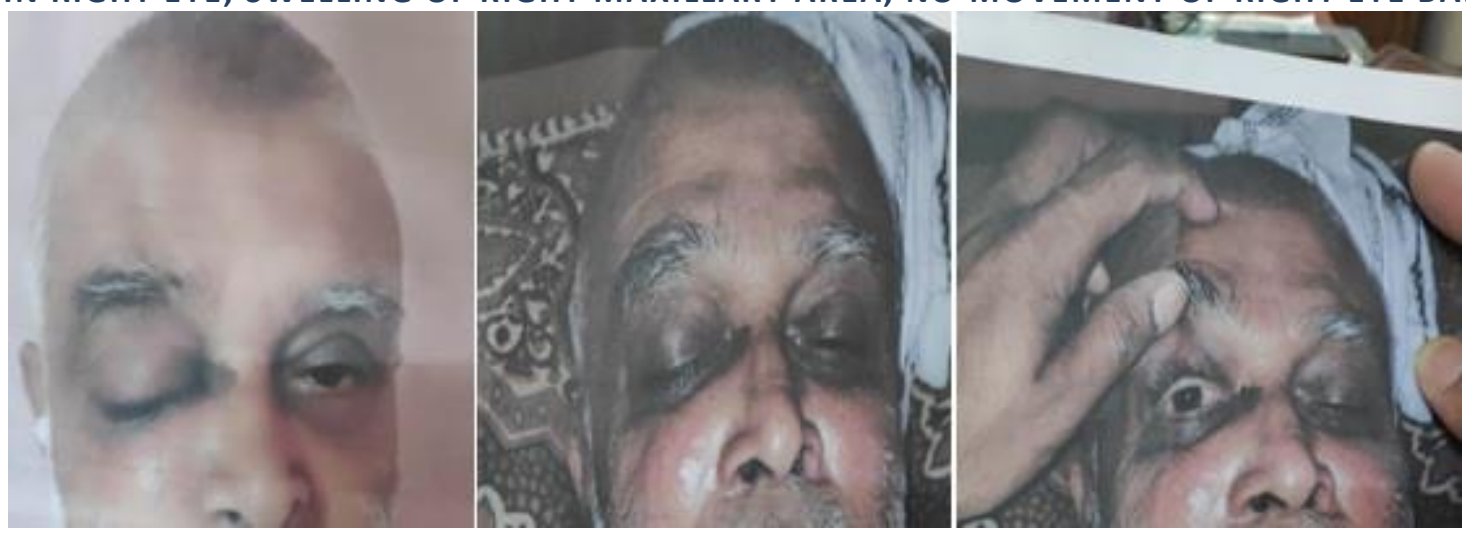

FIGURE 2 TIMELINE OF THE EVENTS AND DEPICTION OF DELAYS.

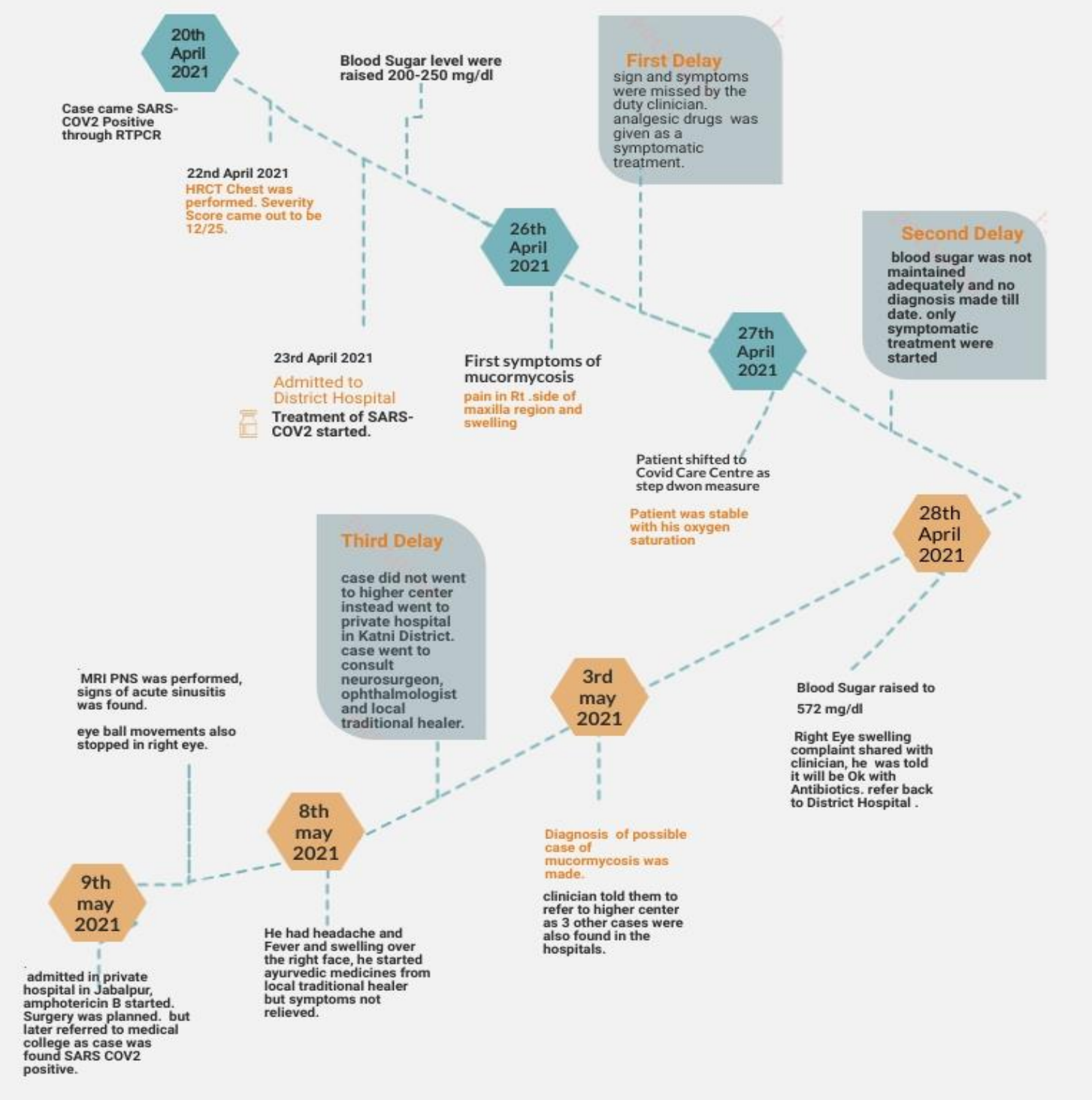

